Rabaska

Revue d'ethnologie de l'Amérique française

\title{
« Le Flambeau d'amour » au Canada : conservation et innovation
}

\section{Bárbara Fernández Taviel de Andrade}

Volume 2, 2004

URI : https://id.erudit.org/iderudit/201645ar

DOI : https://doi.org/10.7202/201645ar

Aller au sommaire du numéro

Éditeur(s)

Société québécoise d'ethnologie

ISSN

1703-7433 (imprimé)

1916-7350 (numérique)

Découvrir la revue

Citer cet article

Fernández Taviel de Andrade, B. (2004). « Le Flambeau d'amour » au Canada : conservation et innovation. Rabaska, 2, 31-50. https://doi.org/10.7202/201645ar d'utilisation que vous pouvez consulter en ligne.

https://apropos.erudit.org/fr/usagers/politique-dutilisation/ 


\title{
« Le Flambeau d'amour » au Canada : conservation et innovation
}

\author{
Bárbara Fernández Taviel de ANDRAde \\ Universidad de Castilla-La Mancha (Espagne)
}

\section{Introduction}

La chanson traditionnelle Le Flambeau d'amour' s'est répandue dans tout le territoire canadien même si elle n'est pas des plus connues. D'une centaine de versions qui composent notre corpus, quarante ont été recueillies en France et en Suisse au cours des enquêtes menées à la fin du $\mathrm{XIX}^{\mathrm{e}}$ siècle et publiées dans des recueils déjà vieux d'un siècle ${ }^{2}$. Les soixante autres, recueillies dans le Nouveau Monde tout au long du XX $\mathrm{XX}^{\mathrm{e}}$ siècle, proviennent de toutes les régions canadiennes non seulement là où les francophones sont majoritaires, mais aussi là où la diaspora des francophones s'est établie : Québec et Nouveau-Brunswick, mais aussi Ontario, Terre-Neuve, Île-duPrince-Édouard, Alberta, Massachusetts, et Louisiane ${ }^{3}$.

Les nombreuses variantes discursives et narratives de cette chanson rendent sa folklorisation hors de doute ${ }^{4}$. Mais ce qui semble le plus remarquable est, d'une part, la conservation dans certaines versions canadiennes de quelques vers de forte allure littéraire alors qu'ils sont absents sur le territoire européen et, d'autre part, la capacité innovatrice de la tradition canadienne, et ce, tant au niveau discursif qu'au niveau narratif, preuve indubitable de la vitalité de la transmission orale dans le Nouveau Monde.

1. Le titre d'amour entre en concurrence avec d'autres titres du genre L'Amant noyé, La Belle dans la tour, La Fille prisonnière, Les Deux Amants... qui expriment un des éléments de la structure narrative, ou bien d'autres du type Qui veut entendre une chanson, C'est une fille de quinze ans, Quel triste jour, etc., tirés tout simplement de l'incipit. Mais en ce qui concerne la fréquence, c'est le titre du Flambeau d'amour qui l'emporte sur les autres. Des folkloristes français tels que Patrice Coirault, Georges Doncieux ou Julien Tiersot sont d'accord pour le nommer ainsi et c'est avec ce titre qu'il est classé dans le Catalogue de la chanson folklorique française de Conrad Laforte (Québec, Les Presses de l'université Laval, 1981, Tome II, Chansons strophiques, $\mathrm{n}^{\circ}$ II-A-10).

2. Deux versions seulement provenant de Bretagne ont été recueillies autour des années soixante du $\mathrm{XX}^{\mathrm{e}}$ siècle.

3. Mes remerciements à madame Carole Saulnier, des Archives de folklore de l'université de Laval, pour m'avoir envoyé la plupart des versions de mon corpus canadien.

4. Voir à ce propos ma thèse de doctorat El mito de Hero y Leandro en la tradición oral europea, Madrid, Universidad Complutense, 1989. 
Les érudits ont depuis longtemps considéré la chanson traditionnelle française Le Flambeau d'amour comme le versant populaire de la légende classique de Héro et Léandre en terres francophones. Nous nous proposons dans ce travail de faire un bref parcours de la trajectoire littéraire de cette légende pour pouvoir ensuite établir sa vie traditionnelle. En second lieu, nous allons comparer quelques vers d'un texte semi-lettré du XVII siècle avec les 100 versions du corpus du Flambeau d'amour, ce qui nous permettra finalement de déterminer la capacité de conservation et d'innovation de la tradition orale canadienne dans le cas précis du Flambeau d'amour. Cependant, loin de nous la prétention de tout dire de Héro et Léandre ou du Flambeau d'amour dans ces quelques pages. Notre seul souhait est de présenter un aperçu de la richesse qu'offre n'importe quelle manifestation de la tradition orale dès qu'on l'analyse en profondeur.

\section{Histoire littéraire et traditionnelle de Héro et Léandre}

Nous ne rappellerons que très brièvement l'histoire tragique de ces deux amants, telle que nous l'a transmise l'Antiquité : Héro est une vierge consacrée au culte de la déesse Aphrodite ; isolée du monde, elle demeure dans une tour dans la ville de Sestes. Lors de la fête d'Aphrodite, déesse de l'amour, Héro fait la connaissance de Léandre, jeune homme habitant la ville d'Abyde, séparée de Sestes par le détroit de l'Hellespont. Ils tombent amoureux l'un de l'autre et conviennent de rendez-vous nocturnes, afin de jouir de l'amour interdit. Pour se rencontrer chaque nuit, Léandre doit traverser le bras de mer qui le sépare de son amie. Celle-ci s'engage à mettre un flambeau tout en haut de la tour afin de guider le courageux amoureux. Les rencontres se succèdent jusqu'au jour où une tempête éteint le brandon et Léandre, faute d'éclairage, $s$ 'enfonce dans le gouffre maritime. Le lendemain, Héro découvre le corps de son amant sur le sable ; ne pouvant échapper au désespoir, elle se précipite du haut de la tour et meurt ${ }^{5}$.

Voyons tout d'abord deux versions « types» du Flambeau d'amour en terres européennes ainsi qu'en terres canadiennes :

5. Héro et Léandre sont les héros d'une légende « localisée » dans deux villes, Sestes et Abyde, qui, peut-on dire, séparent l'Europe et l'Asie de telle façon qu'elles peuvent être considérées frontières naturelles de deux continents. Elles ont été aussi le passage des hommes de l'Antiquité (Alexandre le Grand, par exemple). Le géographe allemand Maiten (« Motivgeschichtliche Untersuchungen zur Sagenforschung III : Hero und Leander », Rheinisches Museum für Philology, vol. 93, 1949, pp. 65-81) suggère que la légende est à l'origine de la construction des phares de l'Antiquité. Par surcroît, l'Hellespont, détroit qui sépare Sestes d'Abyde, possède des courants contraires qui font que la nage est facile dans une direction (de Sestes à Abyde) et très compliquée en sens inverse, ce qui expliquerait la noyade de Léandre. Aujourd'hui encore, si vous visitez Istanbul (l'ancienne Constantinople), le guide touristique vous montrera l'île qui serait prétendûment celle de Léandre. 


\section{Galant noyé}

1. C'est une fille de quinze ans Ou quelque chose davantage Son père l'a mise dans une tour Pour pas qu'on lui ferait l'amour

2. Son cher amant qui était là, Les yeux, les yeux baignés de larmes. Belle, donnez-moi le rendez-vous Belle j'irai vous voir à la tour.

3. Vers les onze heures et les minuit À l'heure que le galant se réveille Il aperçoit flambeau d'amour Allumé au haut de la tour.

4. Flambeau brillant, flambeau d'amour, Il est temps d'aller voir la belle Il n'est plus temps d'y regarder Le beau flambeau est allumé.

5. La mer montait, la mer baissait La mer était en furie.

La mer était z'en grand courroux Il est noyé au pied de la tour.

6. À l'heure que la belle se réveille Regarde en haut, regarde en bas Elle voit son amant au trépas. Lendemain matin z'au point du jour.

7. - Servante, servante, apportez-moi Une belle chemise blanche Pour couvrir le corps de mon amant Celui qui mon cour aime tant.

8. Si j'étais fille en liberté Je m'en irais dans un bocage Je prierais Dieu pour mon amant Celui que mon cœur aime.

9. Avec les pointes de mes ciseaux Je percerais une de mes veines Je laisserais couler mon sang Pour restituer mon amant.

10. Voilà la vie de deux amants Qui ont fait comme la tourterelle Quand ils ont perdu leur mari De branche en branche il se détruit.

(Informateur : M. Mezzara, île de Bréhat, Bretagne, 1894. Collecteur : Ary Renan. Publié par F.-M. Luzel, Revue des traditions populaires, 1894, v. 9, p. 409. Cat. Laforte, A-II-10/104. Titre : Galant noyé)

\section{Le Flambeau d'amour}

1. Ah ! c'est une fille de quinze ans Qui veut se mettre en ménage Ah! père la renferme dans une cour Pour que personne lui fît l'amour.

2. Mon beau galant, si vous voulez, Nous metterons [sic] flambeau pour enseigne Quand le flambeau sera allumé Galant, n'épargnez pas vos pas.

3. Mais quand ça vient sur les minuit, Le flambeau d'amour qui s'allume, Le beau galant s'est habillé À la mer qui s'a [sic] jeté.

4. Voilà la tempête et le vent Voilà la mer qui s'agite.

$\mathrm{Oh}$ ! laissez-moi donc vivre un jour Vous me reprendrez à mon retour

5. Et quand ça vient au matin-jour $\mathrm{Oh}$ ! que la belle fut matinale ! Elle regarda du haut, d'en bas Elle voit son amant au trépas.

6. Oh ! mer, ô mer, cruelle mer, Tu as tué mon Alexandre, Tu as tué l'ami du cour, Tu as tué mon serviteur.

7. Avec la pointe de mes ciseaux Je me percerai une veine Je laisserai couler mon sang Pour faire ressusciter mon amant

8. Père et mère qui n'ont pas voulu Consentir à ce mariage.

Mourons, mourons, mort éternelle, Nous ferons nos noces dans le ciel.

(Informateur : $\mathrm{M}^{\mathrm{mc}}$ A.-M. Arsenault, SaintChrysostôme, Île-du-Prince-Édouard, 1940. Collecteur : J.-T. Le Blanc, ms. no 784 (ms. 9351), Cat. Laforte : II-A-10/7. Titre : Le Flambeau d'amour) 
Voilà deux versions des plus complètes de deux traditions, l'une européenne, de 1892, et l'autre canadienne, de 1940, ayant donc un écart de 50 ans. Les deux ont été recueillies dans des contrées éloignées ou isolées. D'emblée, on y peut reconnaître l'écho de la légende classique contenant les principaux éléments narratifs qui structurent le récit : amours contrariées, flambeau-signal, passage de l'eau et noyade du héros. Le choix de ces deux versions s'explique par le fait qu'elles présentent des éléments tant figuratifs que narratifs absents dans la majorité des autres versions quoiqu'aucune des deux n'en présente la totalité. Toutefois elles permettent au lecteur de se faire une idée des principaux éléments narratifs contenus dans la chanson, et elles peuvent nous servir en tant que point de départ pour notre étude.

Mais comment s'est effectué le transfert du registre littéraire au domaine populaire ? Comment la tradition s'est-elle appropriée la légende classique ? La perméabilité entre les deux couches sociales, lettrée et illettrée, a existé de tout temps ; néanmoins il n'est pas toujours facile ni évident d'établir à quel moment s'est effectué précisément tel ou tel passage de l'une à l'autre. Très souvent, il s'avère impossible de trouver l'origine ou même une petite trace du maillon perdu. Mais commençons par le début et approchons-nous un peu de Héro et Léandre.

Ce n'est pas ici le lieu d'exposer la longue et riche histoire littéraire de la légende de ces deux amants malheureux. Nous rappellerons seulement quelques noms des plus connus : Hérodote et Virgile (qui font seulement des allusions aux amants, ce qui montre qu'ils étaient des héros célèbres dans tout le monde antique), les gloses d'Ovide (Héroïdes, XVII et XVIII), les épigrammes de Martial, etc. Ce qui intéresse tous ces auteurs classiques est surtout la prouesse et la noyade de Léandre. Il faut attendre le Grec Musée (VI ${ }^{\mathrm{e}}$ siècle) et son petit poème de quelque 600 hexamètres pour connaître, plus en détail et avec un goût romanesque, les amours malheureuses des deux jeunes gens, dont nous avons exposé ci-dessus le résumé. Un peu plus tard, et pendant tout le Moyen Âge, la plupart des auteurs grecs sont ignorés (Musée et tant d'autres) et les écrivains, ecclésiastiques pour la plupart, rédigent seulement en latin suivant de près les modèles classiques acceptés par l'Église. Ils reprennent la légende de Héro et Léandre en introduisant un objectif moralisateur : les amours non permises sont punies ; autrement dit, transgresser les normes conduit inexorablement à la mort. Dans tous les cas, par misogynie intrinsèque, c'est toujours Léandre qui est leur centre d'intérêt ${ }^{6}$.

6. Fulgencio Planciades [ $\mathrm{VI}^{\mathrm{e}} \mathrm{s}$.] inaugure la moralisation de la légende en identifiant le nom de Léandre avec lisinandro (dissolution) ce qui sera repris par Baudri de Bourgueil [1046-1130]. Philippe de Vitry [vers 1260-1361], écrivant déjà en français, continue cette tradition moralisante en y ajoutant l'argument mysogine de la culpabilité de Héro dans la mort de son amant : "Leandre dissolucion / Donne, qui met s'entencion / En fols amour, en fols arsure / Amoit Héro: ce fu luxure [...] / L'eschaufement et l'ordure / Naist du sixte membre de femme [...] "). 


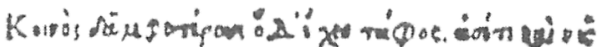

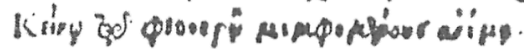

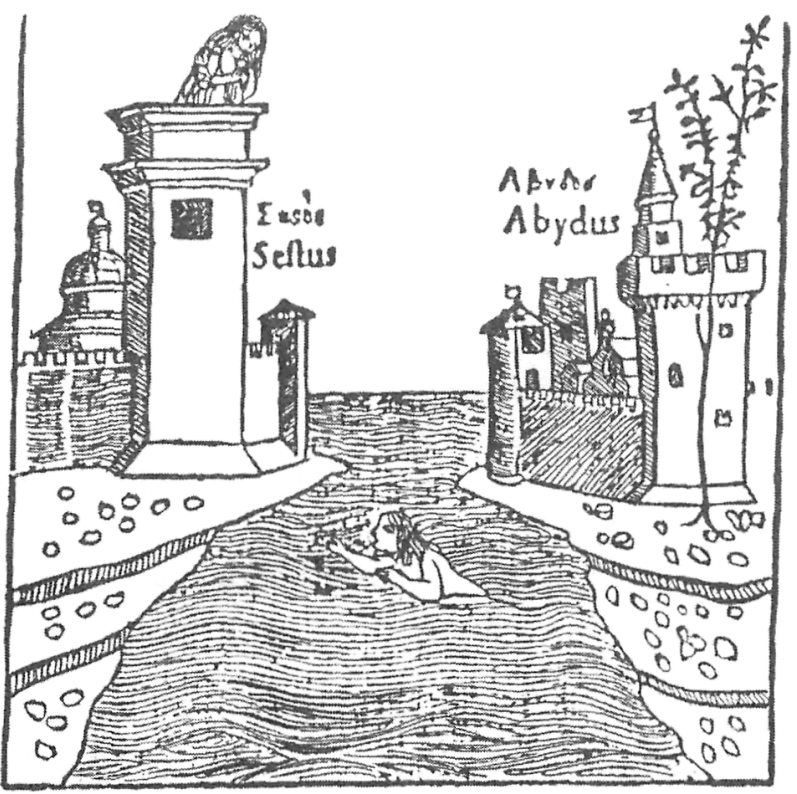

Et puis la Renaissance arriva et ce fut la découverte d'écrivains grecs, Musée y compris. Son petit poème inaugurera l'imprimerie à caractères grecs dans plusieurs pays européens ${ }^{7}$, puis il deviendra par la suite un des « devoirs » obligés de traduction du grec dans les écoles (celle de Clément Marot en 1541 sera la première traduction en français), fait qui le convertira en un des poèmes des plus connus de toute la gent cultivée. Dorénavant, maints poètes de la Renaissance se serviront de la légende de Héro et Léandre comme référence d'exercice de composition poétique. Que le sujet fût déjà très connu et très exploité pendant les XVIe et XVII ${ }^{\mathrm{e}}$ siècles, nous pouvons le déduire par le traitement burlesque qui en est fait : dans le territoire français, par exemple, Rabelais et Scarron - l'un en y faisant à peine allusion et l'autre lui consacrant quelque 600 octosyllabes - montraient par le biais de la parodie à quel point le sujet était déjà traité à satiété. Puis viendront encore des « poètes » de deuxième et troisième rang qui y puiseront leur inspiration et leur verbe poétique. Toujours étant que la noyade de Léandre reste assurément leur centre d'intérêt, tandis que le sacrifice de Héro, si jamais il est mentionné, ne l'est que d'une manière rapide, presque évasive.

7. Il parut pour la première fois à Venise vers 1494, chez Alde l'ancien ; en France, il le fut au commencement de 1507 par Gilles Gourmont, qui semble être le premier à éditer le caractère grec. Par la suite, les éditions du poème de Musée vont se multiplier dans toute l'Europe occidentale. 
Les temps qui suivirent, peu adonnés à la poésie, assistent cependant à un développement d'une modalité poétique populaire qui ne s'est jamais tue durant les siècles précédents : ce sont les chansons de rues, telles que celles du Pont-Neuf, mais aussi les romances à mi-chemin entre la chanson folklorique et la chanson cultivée. C'est justement dans ce courant que l'on doit placer le Sieur de Saint-Amour, dont la chanson se rapportant à Héro et Léandre nous intéresse particulièrement. Absent de la liste d'auteurs réputés, le Sieur de Saint Amour a probablement été, comme tant d'autres amateurs de poésie qui n'ont pas atteint à la célébrité, connu dans les couches populaires grâce à ces livrets divulgués par le circuit du colportage ${ }^{8}$. En effet, la Chanson tirée des Métamorphoses d'Ovide est incluse dans un de ces nombreux recueils qui ont proliféré pendant les $\mathrm{XVII}{ }^{e}$ et $\mathrm{XVIII}{ }^{e}$ siècles et qui forment une grande partie de la Bibliothèque bleue que les colporteurs ont été chargés de distribuer dans le milieu rural jusqu'au milieu du XIX ${ }^{\mathrm{e}}$ siècle.

La collection publiée par le Sieur de Saint-Amour a comme titre Le Trésor des plus belles chansons et airs de cour, tant pastorales que musicales, propres $\grave{a}$ danser et à jouer sur toutes sortes d'instruments. C'est un mélange de sujets sans aucun critère classificateur et la preuve en est que la chanson concernant les amours malheureuses de Héro et Léandre est placée entre deux chansons joyeuses. Sans doute, le recueil de Saint-Amour put-il bénéficier de la popularité de ces petits livrets, mais nous ne connaîtrons jamais la portée de sa divulgation, bien qu'il soit fort probable que sa «Chanson tirée des Métamorphoses d'Ovide $»^{9}$ ait put atteindre de nombreux points du sol européen français ainsi que du territoire d'outre-mer. Mais voyons le texte de Saint-Amour et essayons d'y voir les éléments coïncidant avec le Flambeau d'amour :

8. Parallèlement à cette histoire littéraire, on pourrait parler d'une histoire traditionnelle en Europe de la légende de Héro et Léandre. En effet d'éminents philologues et érudits ont cru voir dans les légendes saintes du XIII ${ }^{e}$ siècle (Gauthier de Coincy en France et Gonzalo de Berceo en Espagne) et dans les légendes localisées dans plusieurs pays européens (Espagne, France, Portugal, Ukraine, Yougoslavie...), ainsi que dans certaines chansons traditionnelles (Allemagne, Pays scandinaves, judéo-espagnols de la diaspora, Piémont, France, Suisse, Catalogne, Canada...) un écho évident du thème légendaire classique. Mais qu'avaient en commun toutes ces manifestations tant littéraires que traditionnelles? C'est que, pour la plupart, on pouvait affirmer qu'elles contenaient un noyau constitué par deux motifs (les amours contrariées et le passage de l'eau), auxquels on pouvait en ajouter un troisième (l'union au-delà de la mort), et deux figures fixes (la mer et le flambeau) auxquelles on pouvait aussi en ajouter une troisième (la tour) exprimant la clôture et l'isolement de la fille. Voir dans ma thèse de doctorat, op.cit., le développement de l'analyse des motifs et figures structurants du mythe.

9. Ce qui est d'ailleurs faux, car ce sont dans les Héroïdes d'Ovide que sont chantées les amours de Héro et Léandre, et non pas dans les Métamorphoses, ce qui prouve encore une fois le caractère semilettré de l'auteur. C'est une des raisons pour lesquelles $j$ 'ai maintenu la graphie originale respectant les fautes d'orthographe. En outre, j'ai souligné les vers qui me semblaient les plus proches de la tradition orale conservées tant en Europe qu'au Canada (Catalogue Laforte II-A-10/92). 
Chanson tirée des Métamorphoses d'Ovide

1. Léandre étoit dessus le bord,

2. De l'Helespont enflé d'Orages,

3. Consultat l'amour \& la mort,

4. Dont il adoroit les images,

5. Pendant que ses desirs passoient

6. Les ondes aussi les menaçoient.

7. Son Soleil étoit sur la tour

8. Qui brûloit d'une telle flamme

9. Lui montrant un flambeau d'amour:

10. Qui le convoie à voir sa Dame ;

11. Soudain qu'il le vit flamboyer

12. Courageux se jette dans la mer.

13. La mer enflée de courroux

14. Lui fait souffrir de grandes peines

15. Lui montrant la mort à tout coups

16. Au bruit des vagues inhumaines :

17. Helas : ondes, si l'amitié

18. Vous touche, ayez de moi pitié,

19. Neptune, grand Dieu de la mer,

20. Vous qui commandez ce rivage

21. Faites ces ondes un peu calmer

22. Helas : gardez-moi de naufrage

23. Permetez que j'aille à la tour,

24. Et me submergez au retour.

25. L'Onde farouche le poursuit ;

26. Et d'une trop cruelle rage,

27. Le retient, le lâche, \& le suit

28. Puis le rend mort sur le rivage

29. Et arrivant l'aube du jour

30. Elle le vit mort sous la tour.

31. Quand elle vit dessous la tour,

32. Le corps de son amy Léandre

33. Privé d'âme, \& que son amour

34. En tel état l'avoit fait rendre

35. O mort! Dit-elle en soûpirant

36. Mets fin à mon cruel tourment
37. Cruel Monarque de la mer,

38. Quel profit t'apporte la perte,

39. D'un amant rempli d'aimer,

40. Comme un genereux Milicerte.

41. Pourquoi donc tout pâle \& transi

42. Privé d'espoir l'a mis ici.

43. Tu devrois perdre ces Vaisseaux

44. Qui font quelque resistance

45. Non pas submerger dans tes eaux

46. Un corps qui cède à ta puissance

47. Chargé de desir \& d'amou

48. D'arriver jusques à ma tour

49. Si dans tes flots cruellement

50. Tu l'as livré à la mort blême

51. Un fleuve qui feroit bien autant

52. Si feroit bien un ruisseau même

53. Et tu as privé d'un beau jour

54. Un amant qui n'étoit qu'amour.

55. O ciel cruel! Ô ciel trompeur :

56. Que t'avoit fait mon cher Leandre?

57. Tu lui ravis l'âme et le cour

58. Et maintenant me le viens rendre

59. Quand elle eut pleuré son trepas

60. Se jetta de la tour en bas.

61. Ainsi cette amante disoit,

62. En batant ainsi sa poitrine

63. Quand la douleur qui le pressoit

64. La fit jetter en la marine

65. Où elle finit à l'instant

66. Ses pleurs \& son cruel tourment.

67. A la mort de ces deux amans

68. Trépassez au bord du rivage

69. A la merci de tous les vents

70. qui sont cause de leur naufrage

71. On a pû voir comme l'amour

72. Avec la mort fait son séjour. 
Le poème de Saint-Amour décrit la prouesse de Léandre, sa mort, puis la plainte de Héro et son suicide. Nous nous sommes permis de souligner quelques vers auxquels nous accorderons une attention spéciale et sur lesquels nous aurons l'occasion de revenir. Le poème présente Léandre sur le point de plonger, ce qu'il fait dès qu'il voit flamboyer le brandon de sa dame. Les vers 12 à 28 décrivent le nageur se débattant sans succès contre les flots et priant le dieu Neptune de l'épargner à l'aller et de le prendre au retour, vers qui évoquent fortement l'épigramme de Martial et sur lesquels nous reviendrons. Cependant, ce qui nous semble tout à fait inhabituel dans la tradition littéraire, c'est l'importance accordée à la plainte de Héro et à la mort de celle-ci : presque une quarantaine de vers lui sont consacrés, du vers 29 jusqu'au vers 66 , ce qui constitue presque la moitié du poème.

Qu'il s'agisse ou non de l'antécédent littéraire (ou texte "préfolklorique » comme l'appellerait Coirault) de la chanson traditionnelle du Flambeau d'amour, nous ne le saurons jamais, à moins que nous ayons la chance d'en trouver la trace dans l'un de ces recueils des XVII ${ }^{e}$ et XVIII ${ }^{e}$ siècles. En revanche, nous refusons, d'ores et déjà, le procédé prôné par des savants tels que Doncieux en France et Barbeau au Canada ${ }^{10}$, d'établir le texte critique (ou " esthétique ») qui serait le résultat du plus grand nombre de variantes les plus fréquentes et de le prendre pour le texte primitif, autrement dit, " la chanson telle qu'elle est sortie pour la première fois de la bouche du chansonnier ${ }^{11} »$. Nous estimons plus plausible de songer à un texte semi-lettré parent de celui de Saint-Amour (si ce n'est pas son texte luimême) qui aurait perdu et gagné des éléments tout au long de sa vie traditionnelle. C'est justement lorsque la tradition se met à l'œuvre que l'on voit chaque interprète s'approprier la chanson et se permettre, inconsciemment, d'éliminer des éléments ou d'en incorporer de nouveaux, et de faire vivre la variante, ce qui est la véritable caractéristique de la transmission orale.

\section{Méthodologie appliquée}

Nous disposons d'un corpus d'une centaine de versions du Flambeau d'amour ( 38 versions françaises, quatre versions suisses et 60 versions canadiennes) qui ont été groupées suivant des critères de proximité géographique. Il s'agit premièrement d'effectuer la segmentation séquentielle, en reconstruisant le modèle narratif sous-jacent. Ce modèle, qui n'est actualisé dans aucune version, est une abstraction élaborée par induction à partir de toutes les versions du corpus et elle systématise et rétablit l'ordre logico-temporel de l'histoire

10. Georges Doncieux, Le Romancéro populaire de la France, Paris, 1904, pp. 282-284, et Marius Barbeau, Le Rossignol y chante, Ottawa, 1962, pp. 173-174.

11. Doncieux, ibid., p. XXXVII. 
racontée. C'est ce que nous avons fait avec quelque cent versions du corpus de trois traditions, en dépit des deux versions traditionnelles proposées plus haut, qui sont, répétons-le, les plus complètes du corpus. Le tableau présenté ci-dessous reflète exactement la totalité des séquences narratives ainsi que leur fréquence dans les trois traditions francophones du Flambeau d'amour.

SEGMENTATION NARRATIVE

1. Exorde

1.1. Formule extra-narrative

1.2. Mise en abîme

2. Présentation des protagonistes

2.1. De la jeune fille

2.1.1. Âge

2.1.2. Qualités (« avantages »)

2.1.3. Situation amoureuse

2.2. Des deux amants

3. Le père de la fille s'oppose à l'union des jeunes

4. Le garçon exprime sa tristesse

5. Le garçon propose un stratagème de rencontre

5.1. La fille doit se laisser enfermer

5.2. Si la fille ouvre la porte de la tour...

5.2. Si la fille lui dit où se trouve la tour...*

5.3. Alors le garçon lui rendra visite

6. La fille promet de mettre le flambeau-signal

6.1. du début de la traversée*

6.2. de la vigueur de l'amour réciproque*

7. Séparation réalisée

7.1. Le père renferme la fille dans la tour

7.2. La tour est entourée d'eau

8. La nuit arrive

8.1. «Le flambeau d'amour » s'allume

8.2. Le garçon est toujours à terre

8.3. Le garçon s'encourage à se jeter à l'eau

8.4. Le garçon se jette à l'eau

8.5. La tempête se déchaîne

8.6. Le flambeau s'éteint

8.7. Le garçon demande clémence à la mer

8.8. Le garçon se noie

8.9. Le corps reste au pied de la tour

$\begin{array}{ccc}\text { France } & \text { SuIsse } & \text { Canada } \\ \mathrm{X} / 38 & \mathrm{X} / 4 & \mathrm{X} / 60\end{array}$

9

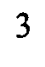

23

52

$\begin{array}{lll}3 & 3 & 19\end{array}$

$6-32$

$2 \quad-\quad 2$

$\begin{array}{lll}35 & 1 & 49\end{array}$

$30 \quad 1 \quad 33$

?

19

1

36

$\begin{array}{lll}18 & 3 & 53\end{array}$

$7 \quad 1 \quad 53$

5 -

$38 \quad 4 \quad 60$

$\begin{array}{lll}35 & 1 & 49\end{array}$

$12 \quad-$

$29 \quad-\quad 53$

$11-51$

- $\quad-\quad 1$

- $\quad-\quad 1$

$2 \quad-\quad 23$

$\begin{array}{lll}6 & 3 & 7\end{array}$

$92 \quad-$

- -5

$7 \quad-\quad 18$

$\begin{array}{lll}7 & 3 & 6\end{array}$ 


$\begin{array}{ccc}\text { France } & \text { SuISSE } & \text { CANADA } \\ \mathrm{X} / 38 & \mathrm{X} / 4 & \mathrm{X} / 60\end{array}$

9. Arrivée du jour

9.1. La fille se penche à la fenêtre

9.2. La fille « regarde du haut en bas »

9.3. "Trouve son amant au trépas"

10. La fille demande de l'aide à la servante

10.1. Et ensevelit le corps de son amant

11. Plainte de la fille

11.1. La fille s'adresse à son amant mort

11.2. Elle invective la mer*

11.3. Elle invective la mort*

11.4. Elle invective ses parents*

11.5. Elle souhaiterait être libre

11.5.1. pour prier*

11.5.2. pour faire comme la tourterelle*

11.6. Elle désire ouvrir ses veines

11.7. Elle promet l'union au-delà

12. Elle perce ses veines et meurt

13. Épilogue

$\begin{array}{ccc}33 & 2 & 46 \\ 17 & 3 & 18 \\ 35 & 3 & 44 \\ 34 & 4 & 46 \\ 1 & - & 1 \\ 1 & 1 & 1 \\ 38 & 4 & 60 \\ 6 & - & 6 \\ 1 & - & 23 \\ 18 & 2 & 4 \\ 3 & - & 14 \\ 20 & - & -2 \\ 4 & - & 2 \\ 11 & 2 & 1 \\ 34 & 1 & 55 \\ - & - & 42 \\ 4 & - & 6 \\ 3 & - & 1\end{array}$

(Remarque : les segments signalés d'un astérisque $\left(^{*}\right)$ sont alternatifs et exclusifs, quoique, au niveau profond, ils puissent avoir la même signification)

L'hypothèse proposant le poème de Saint-Amour comme le précédent littéraire immédiat ou, du moins, une des traces possibles de l'antécédent du Flambeau d'amour est des plus séduisantes. Plusieurs raisons le justifieraient ou du moins l'expliqueraient: d'une part, le grand nombre de vers accordés à la plainte de la fille éplorée et, d'autre part, la proximité discursive du poème de Saint-Amour et des vers les plus significatifs et les mieux conservés par la tradition. Par la suite, la transmission orale aurait fait le reste : prendre ici et là des éléments migratoires (motifs, figures et vers formulaires ${ }^{12}$ ) présents dans la tradition et les incorporer à l'intrigue de la chanson (par exemple, le

12. « Le style formulaire enchâsse dans le discours, au fur et à mesure de son déroulement, et intègre en les y fonctionnalisant, des fragments rythmiques et linguistiques empruntés à d'autres énoncés préexistants, en principe appartenant au même genre, et renvoyant l'auditeur à un univers sémantique qui lui est familier " (Paul Zumthor, Introduction à la poésie orale, Paris, Seuil, 1986, p. 116). Dans d'autres termes s'exprime Rosa Knorringa (Fonction phatique et tradition orale, thèse de doctorat, Amsterdam, 1978) : « Il faut remarquer que les formules vraiment vides de sens sont rares [...] mais elles peuvent garder ce côté décoratif en rapport avec le monde rural. Nous pouvons donc affirmer que les formules sont de véritables constituants des ballades, dans la mesure où leur forme toujours disponible et relativement fixe assure la composition et satisfait le public par l'élément connu, tandis qu'elle véhicule un contenu sémantique, perçu comme propre au genre $»($ p. 28). 
motif du suicide sanglant, la tourterelle, la fille de quinze ans, le père qui enferme la fille, etc.). Mais l'analyse de tout ceci nous éloignerait trop de notre intention présente ; dans une prochaine étude, nous nous pencherons plus en détail sur tous ces aspects concrets en analysant leur conservation ou leur perte dans les diverses branches de la tradition canadienne.

\section{Conservatisme au Canada}

a) Le flambeau d'amour : Le vers 9 de Saint-Amour, « Lui montrant flambeau d'amour ", peut être considéré un lexème-formule, car, non seulement il sert d'intitulé à la chanson (voir note 1), mais aussi il se maintient presque inchangé à l'intérieur de la chanson dans les traditions française et canadienne. Il contient la double charge sémantique de signal de déplacement mais aussi de symbole de l'amour-passion ${ }^{13}$, et les transmetteurs de la tradition en ont pressenti l'importance en le gardant avec zèle (11 occurrences en France et 52 au Canada).

b) Les vers 13 à 24 de Saint-Amour, qui glosent les épigrammes de Martial (40-102 après J.-C.) : Cum peteret et Leandros marmoreus ${ }^{14}$, lesquels ont connu en France une certaine fortune dans la classe littéraire ${ }^{15}$ et qui sont représentés d'une manière irrégulière dans les trois traditions. Voici les vers de Martial :

Le téméraire Léandre se dirigeait vers ses chers amours. Épuisé il était près d'être submergé par les vagues grossissantes quand, dans sa détresse, il adressa, dit-on, cette prière aux flots qui le pressaient : Épargnez-moi à l'aller, ne m'engloutissez qu'à mon retour. (Spectaculorum liber, 25b)

Léandre en marbre : le courageux Léandre clamait entouré des ondes grossissantes : Ondes, submergez-moi à mon retour. (Épigramme, XVI, 181)

Ces vers, absents en Suisse, laissent un certain souvenir dans quelques versions françaises, et on les trouve épars dans la tradition canadienne du Flambeau d'amour. En effet, cinq versions canadiennes seulement conservent les vers qui font écho à Martial, contenus dans le poème de Saint-Amour, où

13. Voir à ce propos notre étude, faite en collaboration avec Michelle Débax, « Le Flambeau pour enseigne " in Littérature orale traditionnelle populaire, Actes du Colloque, 20-22 nov. 1986 (Fondation Calouste Gubelkian, Paris, 1987, pp. 127-140).

14. Cum peteret dulces audax Leandrus amores / Et fessus tumidis iam premeretur aquis / Sic miser instantes adfatus dicitur undas : /-Parcite dum propero, mergite cum rodeo-(Spectaculorum liber, 25b). / Leandros marmoreus / Clamabat tumidis audax Leandros in undis, /-Mergite me fluctus cum rediturus ero- (Épigramme, XVI, 181).

15. L'allusion burlesque faite par Rabelais concerne précisément l'épigramme de Martial : « Tu as (dist Panurge) l'esprit moult limpide et serein, Frère Jam, couillon métropolitain, et parlez pertinemment. C'est ce dont Léandre de Abyde en Asie, nageant par la mer Hellesponte, pour visiter s'amie, Héro, de Seste eu Europe, prioit Neptune et tous les Dieux marins : / Peu au retour me chault d'estre noyé.- / -Si en allant, je suys de vous choyé / « C'est dit-il, qu'il ne vouloit point mourir les couilles pleines ». (François Rabelais, Le Tiers Livre, CEuvres complètes III. (Paris, Garniers-Frères, 1965, p. 515). 
le nageur demande clémence à la mer pour qu'elle accepte d'ajourner sa mort après la rencontre amoureuse :

Ô mer toute remplie de courroux

Tu veux pas qu'alle [sic] voir ma blonde

Laisse-moi aller à la tour

Tu m'y prendras à mon retour. (Gaspé, 1918) ${ }^{16}$

Ô mer toute remplie de courroux

Laissez-moi aller voir ma blonde.

Quittez-moi aller à la tour

Tu m'y prendras seule [sic] à mon retour. (Gaspé, 1948)

ô mer, ô remplie de courroux

Laissez-moi aller voir ma blonde

Laisse-moi aller à la tour

Tu me prendras à mon retour. (Gaspé, 1948) ${ }^{18}$

Voilà la tempête et le vent

Voilà la mer qui s'agite

$\mathrm{Oh}$, laissez-moi donc vivre un jour

Vous me reprendrez à mon retour. (île-du-Prince-Édouard, 1940)

ô mer, ô mer, terrible mer

Laissez-moi aller voir ma mie

Laissez-moi aller à la tour

Tu me reprendras à mon retour. (Alberta, 1921) ${ }^{19}$

Remarquons la provenance des versions : trois proviennent de Gaspé, mais les deux autres proviennent de lieux lointains (Alberta et Île-du-PrinceÉdouard). La comparaison de ces cinq versions, assez semblables, laissent envisager une souche commune, mais on peut aussi déduire que ces vers existaient déjà lorsque la chanson émigra au Canada et qu'ils ont ensuite disparu des autres versions canadiennes. Il est aussi curieux de constater qu'il n'y en a aucune trace dans les versions, d'ailleurs si nombreuses, du Nouveau-Brunswick. Encore plus curieux : seule, une cinquième version canadienne, originaire de Terre-Neuve et recueillie en 1960 , conserve quelques

16. Informateur : François Saint-Laurent (La Tourelle). Collection M. Barbeau, ms. 2120, 1918 ; catalogue Laforte II-A-10/61A.

17. Informateur : Octave Miville (Saint-Joachim, Gaspé). Collection C. Roy, ms. 4905, 1948 ; catalogue Laforte II-A-10/63.

18. Informateur : Léon Collins (Saint-Joachim, Gaspé) Collection C. Roy, ms. 5088, 1948 ; catalogue Laforte II-A-10/64.

19. Informateur : Mme C. Cyr (née Angélique Poirier), Cowley-McLeod, (Alberta). Collection M. Barbeau, ms. 525, 1921; Catalogue Laforte II-A-10/6. 
vers qui rappellent la mer en furie, correspondant aux segments 8.5 et 8.8 de notre segmentation narrative :

La mer était d'un grand courant,

D'un fort courant d'une tempête,

La mer était d'un fort courant,

A jeté l'amant à la tour. (Loretto, Terre Neuve, 1960) ${ }^{20}$

Ce qui nous laisserait supposer que les vers épigrammatiques de Martial y étaient aussi présents. Par surcroît, les quelques autres versions françaises suivantes, elles aussi, ont gardé la trace littéraire de la mer en furie... :

La mer flottant l'a enlevé

Parmi ses eaux, parmi ses ondes

La mer était z'en grand courroux

L'envoya mort au pied de la tour. (Franche-Comté, 1841 et 1924) ${ }^{21}$

La mer montait, la mer baissait,

La mer était en furie.

La mer était $z$ 'en grand courroux,

Il est noyé au pied de la tour. (Bretagne, 1894) $)^{22}$

La mer farouche l'emporté [sic]

Contre les flambs [sic], parmi les ondes

La mer farouche l'emporté [sic]

Devant sa mie s'est arrêté. (Auvergne, 1878) ${ }^{23}$

La mer farouche l'engloutit

Le renversa parmi les ondes

Et le porta de tour en tour

Jusqu'à la porte de la tour. (Périgord, 1912) ) $^{24}$

Ces vers, situés exactement à la bonne place dans l'intrigue narrative, nous permettent d'aventurer l'hypothèse suivante : les vers glosant l'épigramme de Martial étaient à l'origine de la chanson, mais leur forte allure littéraire a aidé à leur élimination et seules quelques versions canadiennes, minoritaires et très conservatrices, les ont gardés, tandis que d'autres, françaises, aussi minoritaires, en ont gardé seulement le souvenir à l'intérieur d'autres couplets proches.

20. Informatrice : Mme Joséphine Costard (Loretto, Terre-Neuve). Collection Peacock, PEA $181.1109,1960$; catalogue Laforte V-A-10/87.

21. A. Marquiset, Statistique historique de l'Arrondissement du Dôle, p. 42 ; catalogue Laforte IIA-10/108 A-B.

22. Informateur : M. Mezzara (île de Bréhat, Côtes-du-Nord, Bretagne). Collecteur: Ary Renan (F.-M. Luzel, Revue des traditions populaires, 9, 1894, 409) ; catalogue Laforte II-A-10/104.

23. V. Smith, "Vieilles chansons du Velay et du Forez», in Romania 7, 1878, $82 \mathrm{n}^{\circ} \mathrm{XXX}$ (Fraisse, Auvergne).

24. S. Trébucq, La Chanson populaire et la vie rurale des Pyrénées à la Vendée, Bordeaux, Féret \& Fils, 1912, t. I, p. 196; catalogue Laforte II-A-10/113. 
c) La conservation du nom "Alexandre 》: Rappelons le vers 56 de Saint-Amour : «Que t'avoit fait mon cher Léandre? ». Il faut dire tout d'abord que dans la tradition européenne du Flambeau d'amour, la trace du nom du héros s'est complètement effacée. C'est pourquoi le fait de trouver si présent et si répandu dans la tradition canadienne le nom d'« Alexandre », qui se rapproche certainement du nom grec de Léandre, est pour tout dire surprenant. En effet, dans 36 des 60 versions canadiennes on trouve, quasiment inchangé, le vers « Tu as tué/noyé mon Alexandre ${ }^{25}$ ».

Plus surprenante encore est la conservation des vers qui suivent le nom de Léandre (vers 57 et 58) de Saint-Amour : «Tu lui ravis l'âme et le cœur. Et maintenant me le viens rendre " qui se retrouvent presque tels quels dans la tradition canadienne (avec quelques variantes discursives concernant le verbe : ravi, sorti, ôté, séparé, arraché, tiré, pris...) et qui nous permettent de le rapprocher des versions recueillies dans le Nivernais ( +18 variantes) qui sont les seules de France possédant ce vers :

Tu lui as ravi l'âme du corps

Et à présent le voilà mort

... et qui rappellent fort ces autres retrouvées dans la tradition canadienne : " Tu y as ravi l'âme du corps », alternant avec ces autres variantes du type "Tu m'arraches l'âme et le cœur » ou " Tu m'y as mis la mort au cœur », ainsi que ceux se référant à la deuxième partie : «Et maintenant, le voilà mort » ou «Et à présent tu me le rends mort », qui conservent presque mot à mot ceux de Saint-Amour et où l'on voit se mettre en œuvre le travail de la tradition, changeant le nom, choisissant ici et là les mots qui lui conviennent.

\section{Innovation et tendances de la tradition canadienne}

Parallèlement à cette tendance conservatrice, nous trouvons la tendance inverse, c'est-à-dire la capacité innovatrice et régénératrice propre à tout organisme vivant, ce qui nous montre également la vitalité indubitable de la tradition orale canadienne. Celle-ci est très évidente dans les segments concernant la plainte de Héro lorsqu'elle découvre le corps mort de son amant :

a) La plainte de la fille :

En effet, déjà longue dans le poème de Saint-Amour (41 vers, donc plus de la moitié du poème), cette plainte subit des changements intéressants dans la tradition orale. Rappelons quelques vers de Saint-Amour qui insistaient sur le destinataire de la plainte : 35 . ( $\mathrm{O}$ mort ! dit elle en soupirant / 36. Mets

25. L'existence du nom Leandro dans la tradition piémontaise, dont le parallélisme narratif et discursif avec la tradition française est manifeste, ainsi que dans la tradition catalane de la légende prouverait d'une certaine manière la préexistence du nom dans la tradition française. Voir C. Nigra, Canti popolare del Piemonte, Torino, Loescher, 1888. Voir aussi M. Débax et B. Fernández, « La Légende de Héro et Léandre dans la tradition orale catalane », in Carrutxa. Fulls de treball 1, 1992, pp. 39-86. 
fin à mon cruel tourment / 37. Cruel Monarque de la mer... 55. O ciel cruel ! Ô ciel trompeur. )

Nous avons donc un destinataire triple de la plainte de la fille : la mort, le dieu de la mer et le ciel trompeur, tous les trois caractérisés par leur cruauté. Un nombre réduit de versions ( 2 en France et 2 en Suisse) présentent la fille malheureuse se lamentant de la souffrance causée par l'amour ("Cruelle chose que d'aimer /Hier soir j'avais un amant/Je n'en ai plus présentement »). Mais, pour la plupart des versions recueillies, la plainte est dirigée à un destinataire précis qui, cette fois aussi, est triple : a) à la mort : "Ô mort, cruelle mort / Que tu me causes de peine », variante ayant plus d'occurrences en Europe (17 en France et 1 en Suisse) qu'au Canada (seulement 1 occurrence); b) à la mer : " Ô mer, cruelle mer. Toute remplie de courroux » où la fréquence d'occurrences est inversée, plus nombreuses au Canada (24 occurrences) qu'en Europe (1 occurrence) ; c) à ses parents : «Ô mère, ô cruelle mère, père malfaisant ", où le glissement sémantique par les homophones 《 mer » > « mère » aboutit à > « père », ce qui entre dans la logique narrative, puisque c'était le père, en définitive, qui était le responsable de la séparation des jeunes amants. Ainsi, si en Europe nous retrouvons seulement 3 occurrences de cette variante, au Canada on en trouve jusqu'à 14 .

Nous constatons donc que, si en Europe, la jeune fille se limite à se plaindre d'un amour malheureux, on assiste à une intensification graduelle de la culpabilité de celui, celle ou ceux qui ont provoqué la mort de l'amant, beaucoup plus perceptible au Canada. De l'insatisfaction de l'amour sans objet et la haine envers la mort elle-même, on dérive vers l'agent qui l'a causée : d'abord, la mer qui conserve le fatum de la tragédie grecque, et puis la mère, dont l'identité homophonique est à l'origine du glissement sémantique. Parfois l'équivoque n'est due qu'à l'action du transcripteur, ce qui peut être considéré déjà comme significatif ; d'autres fois c'est l'apparition du pronom possessif « ma » qui nous permet de déterminer que c'est bien de la « mère » et non de la « mer » dont il s'agit. Mais qu'est-ce que vient faire ici la mère ? N'était-ce pas le père qui avait séparé les deux amants et causé, en dernière instance, la mort du galant? Solution logique : on rend coresponsables les deux parents (" Ô père et mère, vous êtes bourreaux », ou " Cruel père, indigne mère $»)$, ce qui paraît plus compréhensible pour une société moderne. Seulement dans trois versions québécoises, des plus récentes d'ailleurs, la fille s'adresse à son père qu'elle qualifie de « cruel, plein de courroux et sans honneur ». Nous verrons plus tard comment le dieu de la mer et le ciel trompeur de Saint-Amour seront repris dans la tradition orale canadienne tout en renversant le sens négatif et en les transformant en « Dieu éternel », complice et juge suprême des " noces dans le ciel ». 
b) Le sacrifice sanglant : un tableau populaire

La Héro classique se précipitait dans le vide ; Saint-Amour reprend cet acte dans un seul vers : "Se jetta* de la tour en bas » qui, d'une certaine manière, rappelle ce vers formulaire « Regarde du haut en bas », très fréquent dans les trois traditions avec de faibles variantes. Curieusement, les trois traditions sont unanimes à refuser cette mort classique et elles la changent pour une autre plus « sanguinaire » et plus en accord avec le goût populaire. En effet, dans les trois traditions, est très présent ce couplet où l'héroïne désire consommer le sacrifice en ouvrant ses veines, beaucoup plus mélodramatique et prosaïque ( 34 en France, 1 en Suisse, et 55 au Canada). Il faut toutefois observer que, dans la tradition française, la fille exprime aussi le souhait de liberté ( 20 occurrences) pour faire comme la tourterelle (qui non seulement présente l'intérêt symbolique de la veuve inconsolable et fidèle, mais aussi le fait d'être un oiseau qui " vole ", ce qui, dans un certain sens, reprendrait le geste suicidaire de la Héro classique) tandis qu'en terre canadienne son désespoir la mène à ne désirer que la mort. Nous assistons donc à la perte du symbolisme de la hauteur et de la verticalité que véhiculait la « tour »; certes, on pourrait dire que, tant qu'elle est en haut, elle participe de la divinité d'Aphrodite à qui elle est consacrée ; descendre de cette hauteur suppose pour Héro la perte des privilèges divins d'une part, mais d'autre part cela la rend humaine à part entière, donc mortelle. C'est seulement dans la mort qu'elle peut accomplir l'union avec son amoureux Léandre pour toute l'éternité.

Mais la tradition orale nous propose une nouvelle interprétation symbolique de l'histoire : si, dans la légende classique, les deux amants avaient pu avoir des rapports sexuels continus pendant tout l'été - puisque Léandre accomplit sa double transgression chaque nuit, traversant la limite marine et touchant la vierge consacrée -, dans la chanson traditionnelle tout se passe autrement ; en effet, dès sa première traversée, le garçon se noie, et il ne peut donc pas atteindre ni le flambeau ni la belle. Il échoue dans l'entreprise de rendre femme celle qui l'attend. Par son sacrifice sanglant ${ }^{26}$, la fille symbolise l'union charnelle avec son amant : en prenant le couteau ou les ciseaux métonymie de l'épée, métaphore du membre viril -, elle désire laisser couler son sang féminin et " racheter » l'incompétence et l'impuissance de son amant.

26. Le suicide est permis et très fréquent dans le monde classique ; le suicide le plus digne pour les hommes était celui avec l'épée (comme un guerrier), mais le poison était aussi recommandé en cas de tortures imminentes. Les femmes pouvaient elles aussi recourir au poison (ex. : Cléopâtre), mais c'était la précipitation dans le vide qui était le plus courant. Le suicide sanglant était donc privatif et presque un privilège masculin. 
Seulement dans quatre versions françaises, le désir de s'ouvrir les veines est suivi de l'acte ; elles se terminent avec l'" épilogue ", où le narrateur prend la parole pour accuser les deux parents de la fille et les rendre responsables de la mort de leurs enfants ( « Père et mère avaricieux / Vous qui empêchez les alliances / Avez-vous gagné de l'argent / Pour enterrer ces deux enfants $» ?$ ? Bretagne, 1892). Mais la plupart des versions européennes présentent une fin ouverte à l'interprétation de l'auditeur : le désir de mourir s'exprime en temps conditionnel quoique les derniers mots de la plainte permettent de conjecturer une suite malheureuse.

\section{c) L'appel au Dieu éternel}

En ce qui concerne la tradition canadienne, la fin de la chanson se présente tout autrement et on constate que les Canadiens français ont trouvé une solution qui peut être interprétée comme étant dictée par la forte empreinte de leur catholicisme : même s'ils ont conservé le couplet où la jeune fille souhaite ouvrir ses veines, ils en ont incorporé un dernier où la jeune fille fait appel à Dieu, le père éternel, comme contrepoint au cruel père terrestre, pour le rendre témoin et juge de leurs noces, ce qui se traduit dans des formules telles que :

Mourons, mourons, pour l'éternel

Nous ferons nos noces dans le ciel.

Des 41 versions qui possèdent ces vers, 23 présentent « pour l'éternel», 1 « éternité », 4 seulement « père éternel » et 5 « mort éternelle » (qui, d'une certaine manière, reprend l'idée primitive de Musée : « éternellement jusque dans l'abyme de la mort $"$ ).

Donc, d'une part, on peut supposer que le fait suicidaire s'accomplit, ce qui est résolument contre la doctrine chrétienne, mais, d'autre part, la fille demande à Dieu sa complicité et sa bienveillance en acceptant de consacrer leur union dans le ciel, ce qui disculperait la fille et justifierait son suicide.

\section{En guise de conclusion}

Cette vigueur surprenante de la tradition orale canadienne entraîne, nous l'avons déjà dit, la création constante de modifications, c'est-à-dire de variantes. À ce que nous venons de dire, s'ajoutent les vers formulaires qui, eux, expriment le mieux les deux tendances antagoniques de la tradition moderne du Canada : conservation et innovation, caractéristiques d'une tradition menacée et vivante en même temps. Quelques vers formulaires fondamentaux tels que « Flambeau d'amour », " Regarde de haut en bas », "Avec la pointe de mes ciseaux / je me percerais une veine », "Quand ça vint sur la minuit » se maintiennent très vivaces tout en introduisant de nombreuses variantes discursives - qui n'affectent pas leur fonction substantive, 
adjective ou adverbiale - lesquelles nous permettent d'assurer la fidélité saisissante et la double tendance de la tradition orale canadienne.

Mais, peut-être, ce qui nous semble le plus caractéristique de la tradition canadienne par rapport à l'européenne, c'est l'actualisation idéologique et la très probable banalisation de la signification profonde de la chanson. En effet, cette vitalité va de pair avec une tendance « actualisatrice » qui, parfois, peut aller au détriment de la portée symbolique inhérente à la signification profonde et la banaliser à l'extrême. Tel est le cas, par exemple, de la désémantisation de l'élément aquatique - essentiel dans la constitution du mythe - dont la disparition se trouvait embryonnaire dans les versions européennes. L'obstacle que doit surmonter le courageux amant s'est réduit au franchissement d'une simple porte, ce qui suppose la perte de tout le symbolisme de la figure de l'eau. Cela entraîne un changement dans la signification profonde, de telle manière que la transgression de la limite exprimée par le passage de l'eau disparaît complètement. Pareille banalisation s'opère en ce qui concerne l'élément contraire, le feu, consubstantiel aussi à la légende classique et à la structuration du mythe. Il peut devenir un simple objet d'illumination, tel un lampadaire, réduisant à l'extrême sa richesse sémantique, et ayant comme conséquence la perte non seulement de la connotation érotique véhiculée par le feu-passion, mais aussi de sa valeur dénotative en tant que signe complice des deux amoureux.

La dernière version recueillie à Sudbury en 2002 est très illustrative quant à la désémantisation progressive des éléments symboliques du Flambeau d'amour ${ }^{27}$ :
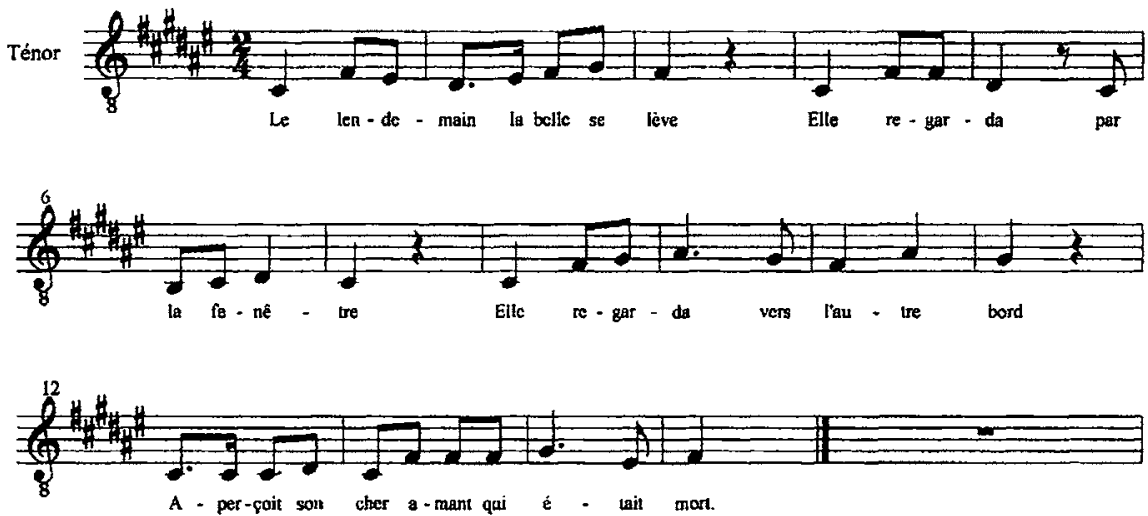

27. Cette version a été chantée à Sudbury le 23 septembre 2002 par M. Gabriel Beaulieu (né en 1940). Tous les travaux d'enquête menés pendant ces vingt dernières années laissaient croire que $L e$ Flambeau d'amour, déjà peu fréquent dans cette région (seulement 4 versions recueillies), avait disparu complètement dans l'actualité. C'est pourquoi, lorsque $M$. Beaulieu nous a chanté sa version, cela nous a émue à un degré extrême. L'enregistrement ainsi que la transcription ont été réalisés par $\mathrm{M}^{\text {me }}$ Olga Beaulieu. Marie-Hélène Pichette en a fait le relevé musical. 
1. C'était une jeun' fille' de quinze ans Grand Dieu qu'elle était amoureuse! Son père lui a fait faire une tour C'était y faire abandonner l'amour.

2. Quand est venu pour s'y mettre en tour Cela lui fit verser des larmes - Ne craignez pas la belle, laissez-vous mettre en tour Là, j'irai vous voir, mais une fois tous les jours.

3. - Venez, amant, venez, venez, Venez, amant, ne craignez rien Quand vous verrez le flambeau s'y allumer N'y craignez pas de vous y approcher.

4. Quand ( $\left.t^{\prime}\right)$ arriva vers les minuits Les flambeaux d' la tour s'allumèrent Le beau galant prit son chapeau pour rejoindre la belle Chercha jusqu'au jour sans y trouver la port' d' la tour.

5. Le lendemain, la belle se lève, Elle regarda par la fenêtre Elle regarda vers l'autre bord Aperçoit son cher amant qui était mort.

6. Maman, maman, ma bonne mère,

$\mathrm{Tu}$ as tué mon Alexandre Tu as tué mon très cher cœur Maintenant je n'aurai plus de bonheur.

7. Avec la pointe de mes ciseaux, Je m'y percerai-t-une veine, Là, j'y verserai tout mon sang Là, j'irai revoir mon fidèle amant.

... où, non seulement le «flambeau d'amour » se multiplie et cesse d'en être un, mais l'amant ne traverse plus les flots et s'endimanche en prenant son chapeau pour rendre visite à son amie.

Nous nous trouvons donc en face du paradoxe que nous avons aussi constaté dans nos enquêtes dans le domaine de la tradition orale espagnole : la vitalité et la vigueur de la tradition orale iraient à l'opposé et au détriment de la qualité poétique de la chanson jusqu'à détruire la capacité d'évoquer des images fortement symboliques et d'aller même à changer la signification profonde du récit. Est-ce le prix que la tradition doit payer pour se maintenir vivante ? Cependant les dépositaires de la tradition ont tout le droit d'adapter le patrimoine traditionnel à leur besoin et à leur goût, et finalement, l'actualisation d'une histoire, si extraordinaire et si sublime qu'elle puisse 
être, implique des changements et des transformations insolites et inespérés qui ne sont pas toujours facteurs d'embellissement mais, du moins, contribuent-ils à la maintenir en vie.

Observe-t-on la même tendance pour toutes les régions canadiennes aujourd'hui ? Certes, nous n'avons travaillé qu'avec des versions recueillies avant 1968, excepté celle de M. Beaulieu, relevée à Sudbury. Il serait très intéressant de trouver d'autres versions plus récentes pour pouvoir corroborer notre hypothèse. On pourrait également continuer la recherche en se servant de cette méthodologie pour établir des groupes de familles de souche québécoise, acadienne, ontarienne, ou du Nouveau-Brunswick. Mais, ce travail reste à faire. 